\title{
Influence Du Mode D'hébergement Et Des Conditions De Vie Des Elèves Sans Tuteurs Sur Le Rendement Scolaire : Cas Du Premier Cycle Du Lycée Moderne De Danané (Côte d'Ivoire)
}

\author{
Agossou Kouakou Mathias, \\ Docteur en Sciences de l'Education \\ Université de Man, Côte d'Ivoire
}

Doi:10.19044/esj.2020.v16n13p320 URL:http://dx.doi.org/10.19044/esj.2020.v16n13p320

\section{Resume}

Cette étude a pour objectif d'analyser la relation entre les conditions de vie des élèves sans tuteurs d'hébergement et leurs rendements scolaires. Les techniques de recherches utilisées sont la recherche documentaire et l'enquête par questionnaire. La méthode d'analyse quantitative a été utilisée sur échantillon stratifié de 530 élèves. Les résultats issus des analyses statistiques montrent que les conditions de vie des élèves basées sur le mode d'hébergement n'a pas d'influence sur le rendement scolaire des élèves en ce qui concerne la satisfaction des besoins en manuels scolaires, l'organisation personnelle de l'élève et l'utilisation de répétiteur par l'élève. En revanche, les conditions de vie des élèves basées sur le mode d'hébergement a une influence sur le rendement scolaire en ce qui concerne la satisfaction des besoins d'alimentation, le sentiment d'être aimé de ses parents et l'idée que se fait l'élève sur la qualité de ses rapports avec son environnement humain immédiat. Ces résultats appellent à des interventions multiformes à plusieurs niveaux du système éducatif ivoirien.

Mots-clés: Conditions de vie-Elève sans tuteurs d'hébergement-Rendement scolaire 


\title{
Influence of The Accommodation and Living Conditions of Students Without Tutors On Academic Performance: Case of The First Cycle from The Danané Modern High School (Ivory Coast)
}

\author{
Agossou Kouakou Mathias, \\ Docteur en Sciences de l'Education \\ Université de Man, Côte d'Ivoire
}

\begin{abstract}
The purpose of this study is to analyze the relationship between the living conditions of students without accommodation tutors and their academic performance. The research techniques used are documentary research and questionnaire surveys. The quantitative analysis method was used on a stratified sample of 530 students. The results from the statistical analyzes show that the living conditions of the students based on the type of accommodation does not influence the academic performance of the students with regard to meeting the needs for textbooks, personal organization and the student's use of a repeater. On the other hand, the living conditions of students based on the type of accommodation has an influence on academic performance with regard to satisfying feeding needs, the feeling of being loved by one's parents and the idea that makes the student about the quality of his relationships with his immediate human environment. These results call for multifaceted interventions on several levels of the Ivorian education system.
\end{abstract}

Keywords: Living conditions-Pupil without accommodation tutors-Academic performance

\section{Introduction : quelques repères théoriques}

Les études portant sur la psychologie de l'adolescence et de l'apprentissage en rapport avec la réussite scolaire sont nombreuses et varient d'un auteur à un autre. (Audas et Willms, 2001 ; Beaumont, 2003 ; Beaumont, Royer, Bertrand et Bowen, 2003 ; Baker, 2006 ; Debarbieux, Anton, Astor et Benbenishty, 2012 ; Guimard, Bacro et Florin, 2014 ; Guimard, Bacro, Florin, Ferrière, Gaudonville et Thanh-Ngo, 2015 et Guimard, Bacro, Florin, Ferrière et Gaudonville, 2017). Aussi, les études sur la lutte contre l'échec scolaire sont complexes et variées. Les causes de la difficulté d'apprendre sont multiples 
(Amigues, et Zerbato-Poudou, 2000 ; Clerget, 2000 ; Kamanzi et Bélanger, 2003 ; Asdih et Gez-M'bembo, 2005). Comme le montre Fiske (1998) dans un aperçu historique de cette question dans les pays occidentaux, notamment en France. Avant la seconde guerre mondiale, plus de la moitié des élèves issus des milieux populaires (paysans et ouvriers) qui fréquentaient pendant plusieurs années l'école primaire abandonnaient souvent sans avoir obtenu de diplôme (le certificat d'études primaires). De même, au secondaire il y avait des situations de redoublement, d'abandon, d'échec au brevet d'études du premier cycle ou au certificat d'aptitudes professionnelles. Mais à l'époque, ces situations n'étaient pas désignées sous le vocable d'échec scolaire et cela, pour deux principales raisons. D'une part, le discours officiel, qui soutenait que la fonction essentielle de l'école était d'assurer la formation de tous les enfants et de leur donner un bagage minimum ( « le niveau du certificat d'études »), ne pouvait admettre de voir l'école accusée de mettre ou de produire des enfants en échec. D'autre part, la situation du marché du travail était telle qu'elle ne marginalisait pas les personnes sans diplôme mais, au contraire, les récupérait très vite à travers différentes styles pédagogiques (Maccoby et Martin, 1983 ; Doré, 1986 ; Lovaas, 1987 ; Dubé, 1996 ; Fuchs et Fuchs,1998 ; Hébert, Boissé et Audet, 1998 ; Flessas et Lussier, 2001 ; L'Abbé et Morin, 2001 ; Goupil, 2004 ; Forget, Schuessler, Paquet et Giroux, 2005 ; Leroi, 2007).

Dans l'après seconde grande guerre, la question de l'échec scolaire prend une signification autre, devenant dans nombre de sociétés une question préoccupante (Lautrey, 1980 ; Blanchard, Casagrande et McCulloch, 1994 ; Duru-Bellat, 2002 ; Boutin, et Daneau, 2004 et Bonnéry, 2008). En effet, le baby boom d'après guerre conduit à la massification scolaire. Selon Bastin et Roosen (1990), plus l'école s'ouvre à tous, plus sont rendus visibles les « exclus » ou les élèves dits en échec. À cela s'ajoute l'emprise des diplômes sur l'entrée dans la vie et l'ascension sociale. L'obtention d'un diplôme ou d'une qualification devient nécessaire pour participer à la vie sociale, notamment professionnelle et espérer un meilleur statut. Autrement dit, la réussite sociale et professionnelle dépend de plus en plus fortement du niveau scolaire, du diplôme obtenu (Grahay, 1996 et Chariot, 2000). Il y a donc une plus grande exigence à l'égard de l'école, entre autres, celle de mettre à la disposition de la société et des instances de production (agricole, industrielle...) une main d'œuvre qualifiée. L'échec devient de moins en moins admis, le retard scolaire, l'abandon ou la non-obtention d'un diplôme pouvant conduire au chômage, à la marginalisation et à l'exclusion sociale (Baker, Dilly, Aupperlee et Patil, 2003; Urdan et Schoenfelder, 2006 ; Koudou, 2006 ; Currie,. Zanotti, Morgan, Currie, de Looze, Roberts, Samdal, Smith et Barnekow, 2012 ; Coudronnière, Bacro, Guimard et Florin, 2015). Comme le démontrent Destexhe, Vandenberghe et Vlaeminck, (2004), les pouvoirs publics dans plusieurs pays 
se mobilisent autour de cette question, engagent des investissements et proposent des solutions politiques et pédagogiques en vue d'enrayer le phénomène. Des budgets particuliers sont alloués pour réformer l'école, des forums et des consultations sur la question sont organisés, il y a création de différents types d'école, formation d'enseignants et autres personnels scolaires, mise en place des pédagogies dites de soutien ou d'éducation scolaire compensatoire, etc. En somme, l'échec scolaire devient peu à peu une question vive, un problème social complexe qui préoccupe un nombre croissant et diversifié d'acteurs sociaux comme les familles, les élèves, les enseignants, les administrateurs scolaires, les gouvernants, les chercheurs et les chercheuses (Corsini et Raymond, 1984 ; Bertrand, 1990 ; Bee, 1997 ; Diehl, Lemerise, Caverly, Ramsay et Roberts, 1998 ; Berndt, Hawkins et Jiao, 1999 ; Braun, 2000 ; Bowen et Desbiens, 2002 ; Bee et Boyd, 2003 ; Crosnoe et Elder, 2004).

En Afrique noire et plus particulièrement en Côte d'Ivoire, la question de l'échec scolaire est également vive et récurrente (Adda, 1997 ; Bih, 1997 ; Dedy, Bih et Koné, 1997 ; Koné, 1998 ; Koudou, 2006 et Kouadio, 2015 et 2017). Elle touche tous les niveaux, du primaire au supérieur et prend plusieurs formes. Par exemple, au primaire, plus du tiers des élèves redoublent leur classe. Au secondaire, plus de $20 \%$ des élèves du premier cycle reprennent une classe, pourcentage qui est plus élevé au deuxième cycle où près de $30 \%$ des élèves sont dans cette situation. Toujours au secondaire, près d'un tiers des élèves, des lycées et collèges confondus, décrochent ou abandonnent en cours de route. L'échec aux examens nationaux est massif. Chaque année au primaire, moins de $30 \%$ des élèves réussissent le concours d'entrée en $6^{\text {ème }}$ qui donne accès au collège. Au secondaire, les taux d'échec aux examens de fin d'études de collège et de lycée dépassent parfois les $80 \%$. Au supérieur, l'échec est aussi considérable, notamment en première année d'études. Par exemple, entre 63 et $76 \%$ des étudiants et étudiantes des facultés seraient concernés chaque année (Ministère de l'éducation Nationale, 2017 et 2018). À ces données statistiques, s'ajoutent les drames que vivent les élèves qui échouent. En quittant l'école sans diplôme, plusieurs se retrouveraient selon Moumouni, (1998) et Noumba, (2008), dans une situation précaire voire d'exclusion, ce qui pourrait les amener à subir n'importe quelle exploitation (Ministère de l'éducation Nationale, 2018). Comme dans les pays occidentaux, la question de l'échec scolaire en Côte d'Ivoire est centrale et comporte aussi, des répercussions importantes sur les élèves (Wang, Haertel et Walberg, 1994 ; Ormrod, 1995 ; Monneret et Marc 1996; Morin, 1996 ; Wentzel, 1998 ; McLoyd, 1998 ; Pianta, 1999 ; Rondeau, Bowen et Bélanger, 1999 ; Willms, 2002 ; Magerotte et Rogé, 2004).Toutefois, l'examen de travaux portant sur les dimensions en relation avec l'échec scolaire, notamment la pratique de confiage scolaire, permet de fournir un éclairage sur divers aspects pouvant 
être associés à l'échec scolaire (Bastin et Roosen, 1990 ; Ballion, 1993 ; Blanchard, Casagrande et McCulloch, 1994 ; Griffaton, 1995 ; Best, 1997 ; Bautier et Rochex, 1998). Au demeurant, les études examinées, même si elles ne traitent pas du sujet tel que libellé dans cette étude, nous apportent beaucoup dans la compréhension du phénomène. Mieux, les différentes études qui se situent dans une perspective déterministe, nous permet de saisir l'objet de l'étude (Lange, 2003 et 2007 ; Millet et Thin, 2005 et 2007 ; Merle, 2005 et 2006).

Cette étude nous renvoie ainsi, à trois notions principales qu'il convient d'examiner. Ce sont l'hébergement, l'élève sans tuteur et le rendement. Pour le premier, selon le Dictionnaire le petit Larousse illustré (2018), hébergement renvoie à la fois à l'action d'héberger et au logement. Mais ce substantif (hébergement) procède du verbe héberger qui signifie, selon la même source, recevoir, loger chez soi. Cette définition du verbe cadre bien avec la situation des élèves. En effet, ils sont en général reçus soit par des personnes physiques (parents biologiques, tuteurs et logeurs), soit par des personnes morales (foyers). Dans tous les cas, l'élève ne loge pas chez lui. Il est reçu à vivre chez autrui, à titre gratuit ou onéreux. Dans cette étude, la notion d'hébergement désigne toutes les modalités par lesquelles l'élève vit sous un toit en vue de poursuivre ses études. Le deuxième, l'élève sans tuteurs, dans une acceptation juridique, le tuteur est celui qui reçoit par une procédure judiciaire, la charge d'assurer l'éducation d'un enfant mineur. Mais dans le cadre scolaire, le tuteur est celui qui abrite sous son toit, un élève qui n'est ni son fils ni sa fille. L'élève sans tuteur est donc, celui qui ne vit pas sous le toit et la responsabilité d'une personne physique adulte. Ainsi définie, la notion pourrait s'appliquer à deux catégories d'élèves seulement (les élèves qui vivent dans un foyer et les élèves qui vivent dans un studio (par euphémisme). Les conditions d'hébergement de ces deux catégories d'élèves, constituent des modalités d'une des variables essentielles de l'étude. Le troisième enfin, la notion de rendement peut se prêter à plusieurs acceptations. Tout dépend du domaine d'intervention. En économie comme en agriculture par exemple, elle s'apparente à la notion de productivité. Mais, c'est le Dictionnaire le petit Larousse illustré (2018), qui l'aborde dans un sens qui rejoint nos préoccupations. Il la définit comme étant le rapport entre le temps que quelqu'un passe à faire un travail, l'effort qu'il fournit et le résultat obtenu. Comme nous le voyons, sous ce rapport, il s'agit de savoir si le résultat du travail est positif ou négatif. Sous l'angle de cette étude, le rendement renvoie aux résultats obtenus par les élèves, à l'issue des évaluations sommatives. L'ensemble pose la question de savoir, à quels résultats peut parvenir un élève qui ne vit pas sous l'autorité d'une personne adulte. À travers cet ensemble, nous comprenons que l'élève (un sujet) utilise la modalité d'hébergement (un moyen) pour produire un rendement (un résultat). Si nous admettons que 
l'hébergement de l'élève peut influencer ses résultats dans un sens comme dans l'autre, nous en déduisons que ces deux éléments sont des variables majeurs de l'article. Or, depuis les années 1990, l'État de Côte d'Ivoire a renoncé à la politique de construction d'établissement à régime d'internat, eu égard à ses difficultés financières. Aujourd'hui, au plan national, l'augmentation exponentielle des effectifs dans le secondaire a obligé des responsables à convertir des dortoirs en salle de classe dans plusieurs établissements. Les différents programmes d'accroissement des capacités d'accueil tels que le projet Banque africaine de développement / Education, les dons Japonais, le plan d'urgence ne prévoient pas non plus de centres d'accueil pour élèves. Quant aux structures décentralisées telles que, les Communes et les Régions, elles cherchent à parer au plus urgent. Très peu d'établissements disposent encore d'internats pour les élèves.

À Danané, la ville ne dispose d'aucun établissement à régime internat. Seuls trois foyers privés tiennent lieu de pensionnat qui reste à désirer. En outre, la demande de logements contraste avec les possibilités réelles qu'offre la ville. La pénurie de logement à atteint des seuils inquiétants. Les populations civiles, potentielles tutrices en logements des élèves, éprouvent d'énormes difficultés à se loger. Des familles des Fonctionnaires ou Agents de l'Etat se regroupent dans des appartements. D'autres, refusant la promiscuité, s'installent dans des localités voisines d'où elles viennent travailler. Une situation qui n'est pas de nature à favoriser l'hospitalité. Des questions émergent de ce constat : Quels rendements peuvent avoir des élèves ayant des problèmes d'hébergement? L'environnement socio-éducatif est-il véritablement déterminant dans la vie des élèves ? Les origines sociales jouent-elles un rôle dans cet environnement ? Quel est l'impact des conditions d'hébergement sur le rendement scolaire ? Comment réduire le retentissement des conditions d'hébergement sur le rendement scolaire ? l'objectif de cet article est d'identifier la nature des relations qui existe entre les conditions de vie et travail des élèves du lycée moderne de Danané d'une part et le rendement scolaire de ceux-ci en vue de susciter des mesures correctives d'autre part. L'hypothèse de cette étude est la suivante: la réussite ou l'échec scolaire est le résultat de la conjugaison de plusieurs facteurs qui sont déterminés par le mode l'hébergement. Les hypothèses opérationnelles de cette étude seront-elles bâties autour de : la satisfaction des besoins en manuels scolaires et en rations alimentaires, l'organisation du travail en dehors des heures de classe et le sentiment d'être aimé part ces parents et de vivre en harmonie avec son environnement humain immédiat. Cet ensemble de préoccupations s'exprimant dans le cadre général des conditions d'hébergement. Quelle est la méthodologie que nous avons utilisée ? 


\section{Méthodologie}

\section{II.1. Site et participants à l'étude}

L'enquête s'est déroulée au Lycée Moderne de Danané (du 04 Avril au 04 juin 2019) qui est un établissement mixte à régime externat à l'ouest de la Côte d'Ivoire. L'échantillon est constitué de 530 élèves du premier cycle $\left(6^{\text {ème }}, 5^{\text {ème }}, 4^{\text {ème }}\right.$ et $\left.3^{\text {ème }}\right)$ âgés de 09 à 15 ans. Si on s'en tient exclusivement au libellé du sujet, le travail porte essentiellement sur deux catégories d'élèves : les élèves pensionnaires de foyers et ceux locataires de studio. Pour conduire le travail de recherche, nous avons identifié les différentes catégories sur lesquelles vont porter l'enquête. Deux sous-groupes ou strates ont été constitué. Ce sont les élèves vivants sous l'autorité de leurs parents biologiques ou vivants chez un tuteur et ceux vivants en pensionnaire d'un foyer ou locataire de studio. Elles sont transversales, relativement aux niveaux concernés. Nous avons choisi ce type d'échantillonnage, parce qu'il convient mieux à l'étude et permet de tenir compte des différentes modalités de la principale variable indépendante de l'étude.

\section{II.2. Instruments de collecte des données}

Les techniques de recherches sont: la recherche documentaire et l'enquête par questionnaire. La recherche documentaire a permis de réunir quelques publications faites par des auteurs déjà connus dans le domaine des sciences de l'éducation. L'enquête par questionnaire porte sur l'identification des enquêté avec 7 items, le mode d'hébergement avec 8 items, l'organisation personnelle de l'élève avec 5 items, l'organisation matérielles de vie avec 7 items et les conditions affectives avec 5 items. Trois types de questions ont été utilisés : des questions ouvertes, fermées et semi ouvertes.

\section{II.3. Méthodes d'analyse des données}

La méthode d'analyse utilisée est quantitative. Deux instruments (statistique et informatique) ont facilité le traitement et l'exploitation des données. Au niveau de la statistique, elle a permis de traduire les données recueillies en sommes, moyennes et proportions. Ce premier niveau de calcul a rendu possible, la présentation des données en tableaux et graphiques. C'est par exemple, avec cet instrument qu'on été quantifié l'échantillon. Il a surtout permis de répondre aux différents problèmes que soulèvent les hypothèses. Croiser les variables pour détecter l'existence d'une relation entre elles en utilisant le test de khi-deux. Au niveau de la méthode informatique, deux ont été essentiels pour la réalisation du masque de saisie, la saisie des données et leur exploitation, nous avons utilisé EPI6 INFO. Pour l'exploitation des données, nous avons recouru également au programme SPSS. Quels sont les résultats obtenus? 


\section{Résultats}

Les résultats s'articulent autour de 11 points suivants: 1-les caractéristiques sociodémographiques des enquêtés ; 2- ; répartitions selon les différents facteurs d'influence sur le rendement scolaire 3-relation entre rendement scolaire et conditions d'hébergement selon la satisfaction des besoins en manuels scolaires; 4-relation entre rendement scolaire et conditions d'hébergement selon la satisfaction des besoins alimentaires ; 5 relation entre rendement scolaire et conditions d'hébergement selon l'organisation de personnelle d'étude ; 6-relation entre rendement scolaire et conditions d'hébergement selon l'utilisation d'un répétiteur ; 7-relation entre rendement scolaire et conditions d'hébergement selon l'affectivité parentale ; 8-relation entre rendement scolaire et conditions d'hébergement selon l'environnement humain immédiat; 9-relation entre rendement scolaire et conditions d'hébergement par niveaux d'études.

\section{III.1 : caractéristiques sociodémographiques des enquêtés}

Graphique $\mathbf{n}^{\circ} \mathbf{1}$ : Répartition des élèves en fonction du sexe

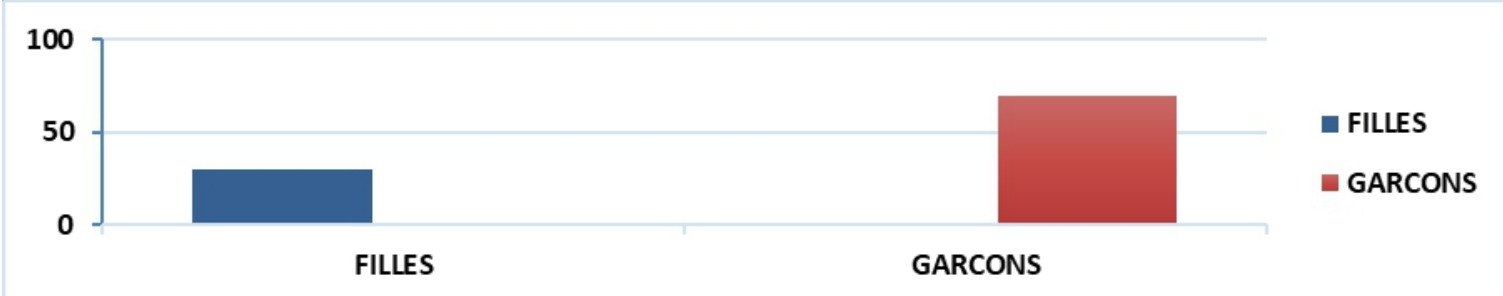

Source : enquête du 04 Avril au 04 juin 2019 au lycée moderne de Danané

Les élèves de sexe masculin constituent la frange la plus importante ; $70 \%$ de l'effectif. Ils représentent un peu plus du double de la proportion des filles. Celles-ci, forment environ $1 / 3$ soit $30 \%$ des élèves. Il convient de noter qu'au niveau des effectifs du premier cycle et de l'effectif général de l'établissement, on observe quasiment les mêmes proportions : Au niveau du premier cycle, $32 \%$ pour les filles, contre $68 \%$ pour les garçons. Au niveau général, $30 \%$ pour les filles et $70 \%$ pour les garçons.

Tableau n ${ }^{\circ}$ 1: répartition des élèves selon le niveau d'études

\begin{tabular}{|l|l|l|}
\hline Classe /Effectif & Effectif & pourcentage \\
\hline Classe de sixième & 114 & 21,50 \\
\hline Classe de cinquième & 144 & 27,16 \\
\hline Classe de quatrième & 139 & 26,22 \\
\hline Classe de troisième & 133 & 25,09 \\
\hline Total & 530 & 100 \\
\hline
\end{tabular}

Source : enquête du 04 Avril au 04 juin 2019 au lycée moderne de Danané

Dans ce tableau, en dehors des classes de sixième qui ont une représentation légèrement inférieure (3 points en moins) à celles des autres, 
chaque niveau d'études est représenté à environ $1 / 4$ de l'échantillon. La répartition des élèves en fonction du niveau d'études est donc globalement équilibrée.

Tableau $\mathbf{n}^{\circ} \mathbf{2}$ : répartition des élèves selon l'origine sociale

\begin{tabular}{|l|l|l|}
\hline Catégorie socioprofessionnel/Effectif & Effectif & pourcentage \\
\hline Enfant de Planteur & 340 & 64,15 \\
\hline Enfant d'Ouvrier & 91 & 17,16 \\
\hline Enfant de Cadre & 73 & 13,77 \\
\hline Enfant de Commerçant & 11 & 02,07 \\
\hline Enfant de Sans-emploi & 15 & 02,83 \\
\hline Total & 530 & 100 \\
\hline
\end{tabular}

Source : enquête du 04 Avril au 04 juin 2019 au lycée moderne de Danané

Les enfants de planteurs sont de loin, la catégorie d'élèves la plus importante. Ils représentent $64,15 \%$ de l'effectif. En revanche, les enfants de commerçants $(02,07 \%)$ et de sans-emploi $(02,83 \%)$ ne sont que des catégories faiblement représentées. Quant aux enfants d'ouvriers et de cadres, ils représentent respectivement $17,16 \%$ et $13,77 \%$ de l'effectif. La zone du Lycée, essentiellement forestière et agricole, semble avoir influencé cette répartition. En effet, la catégorie socioprofessionnelle la plus représentée dans cette localité, est celle des planteurs. Si à cela, s'ajoute le fait de la politique de l'orientation ivoirienne qui consiste à maintenir les élèves plus près de leurs parents, on comprend aisément cette grande différence entre cette catégorie d'enfants et les autres. Ces données, laissent supposer que le lycée est un établissement pour enfants de paysans.

Graphique $\mathbf{n}^{\circ} \mathbf{2}$ : répartition selon la résidence habituelle des parents

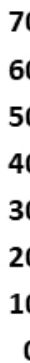

60

50

40

30

20

10

0
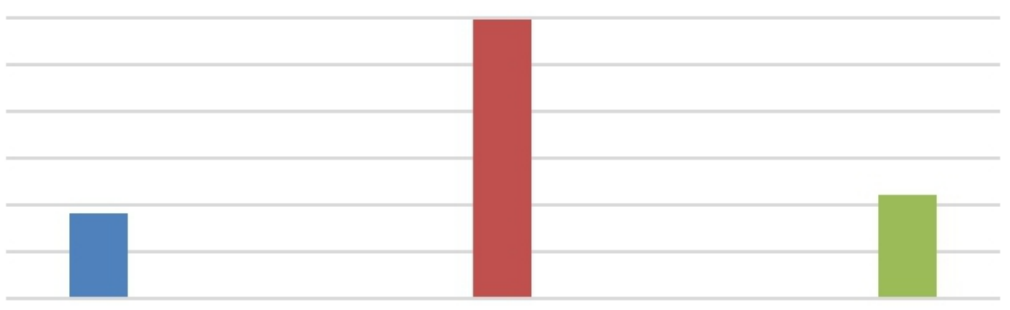

- Dans la ville

Dans le Département

- Hors du Département

Dans la ville

Dans le Département

Hors du Département

Source : enquête du 04 Avril au 04 juin 2019 au lycée moderne de Danané

Dans Ce graphique, les parents d'élèves qui résident dans la ville ne représentent que $18,11 \%$. C'est-à-dire un peu moins d'un parent sur (- de 1/5) vit dans la ville. Un peu plus d'un parent sur cinq (+ de $1 / 5)$, soit $22,07 \%$ vit en dehors de la région d'implantation de l'établissement. Quant aux parents résidant dans la région $(59,81 \%)$, ils donnent un rapport d'environ deux parents sur trois. Une analyse plus fine indique que la plupart des parents 
d'élèves résident habituellement et de façon constante, dans les localités comme les villages et les campements de la ville. Ces parents vivent donc à l'intérieur du département de Danané mais ils ne sont pas des citadins. En ville, on ne compte que très peu de parents d'élèves. Cette situation explique sans doute, la forte propension des élèves à vivre hors de l'autorité des personnes adultes. D'une certaine façon, on peut globalement affirmer que le lycée est surtout ouvert aux enfants dont les parents ont leur résidence principale dans la région.

\section{III.2 : repartions selon les différents facteurs d'influence sur le rendement scolaire \\ III.2.1 : ration alimentaire comme facteur d'influence sur le rendement scolaire}

Tableau $\mathbf{n}^{\circ} \mathbf{3}$ : répartition des élèves selon la ration alimentaire journalière

\begin{tabular}{|l|l|l|}
\hline Nombre de repas par jour/Effectif & Effectif & Pourcentage \\
\hline 1 repas/jour & 97 & 18,30 \\
\hline 2 repas/ jour & 312 & 58,86 \\
\hline 3 repas/jour & 121 & 22,83 \\
\hline Total & 530 & 100 \\
\hline
\end{tabular}

Source : enquête du 04 Avril au 04 juin 2019 au lycée moderne de Danané

Ce tableau indique que la plupart des élèves $(58,86 \%)$ ont une ration alimentaire de deux repas par jour. Un peu plus d'un élève sur cinq $(+$ de $1 / 5$ des élèves), c'est à dire $22,83 \%$ ont une ration alimentaire de trois repas par jour. Enfin, 18,38\% ont un repas par jour. En même temps qu'ils montrent que très peu d'élèves mangent à leur faim, ces chiffres laissent entrevoir une situation loin du drame. En effet, deux repas par jour pour des enfants, généralement issus de familles paysannes et probablement modestes, est une situation courante.

\section{III.2.2 : sentiment d'être aimé de ses parents comme facteur d'influence sur le rendement scolaire \\ Graphique $\mathbf{n}^{\circ} \mathbf{3}$ : répartition en fonction du sentiment d'être aimé de ses parents}

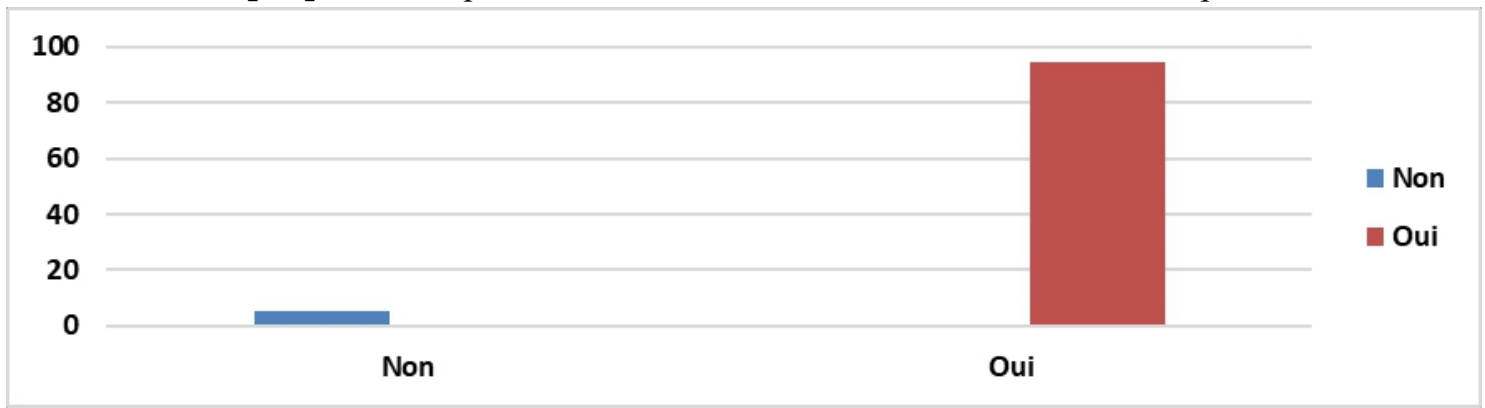

Source : enquête du 04 Avril au 04 juin 2019 au lycée moderne de Danané 
$94,71 \%$ des élèves ont le sentiment d'être aimés de leurs parents. Très peu parmi eux $(05,28 \%)$ pensent le contraire. Ces données, permettent de juger le niveau de discernement des élèves. En effet, elles montreraient que quelles que soient les conditions de vie difficiles, dont ils pourraient par ailleurs rendre leurs parents responsable, les élèves pensent que ces derniers les aiment toujours. Probablement, en retour, ils aiment leurs parents. Le « contrat affectif » n'est donc pas entamé par les vicissitudes de la vie.

\section{III.2.3 : qualité des rapports comme facteur d'influence sur le rendement scolaire}

Tableau $\mathbf{n}^{\circ}$ 4: répartition selon la qualité des rapports

\begin{tabular}{|l|l|l|}
\hline Qualité des Rapports/Effectif & Effectif & Pourcentage \\
\hline Bons & 461 & 86,98 \\
\hline Insatisfaisants & 69 & 13,01 \\
\hline Total & 530 & 100 \\
\hline
\end{tabular}

Source : enquête du 04 Avril au 04 juin 2019 au lycée moderne de Danané

Si l'on déplore le fait qu'il y a quelques élèves $(13,01 \%)$ qui ne s'entendent pas avec leur environnement humain immédiat, il est heureux par contre de constater que environ $87 \%$ des élèves, vivent dans un climat social empreint de cordialité et d'entente avec leurs camarades. Une situation plutôt bonne, quand on connait le rôle de l'élément affectif dans l'équilibre psychologique de l'homme en général et de l'élève en particulier.

\section{III.2.4 : mode d'hébergement comme facteur d'influence sur le rendement scolaire}

Tableau n ${ }^{\circ} 5$ : répartition du mode d'hébergement

\begin{tabular}{|l|l|l|}
\hline Mode d'hébergement/Effectif & Effectif & Pourcentage \\
\hline En famille biologique & 102 & 19,25 \\
\hline Chez un tuteur & 157 & 29,62 \\
\hline Pensionnaire dans un foyer & 25 & 04,71 \\
\hline Locataires d'un Studio & 246 & 46,42 \\
\hline Total & 530 & 100 \\
\hline
\end{tabular}

Source : enquête du 04 Avril au 04 juin 2019 au lycée moderne de Danané

Les élèves vivent majoritairement à $46,42 \%$ dans les studios. Vivre chez un tuteur $(29,62 \%)$ est le deuxième mode d'hébergement des élèves. Vivre en famille biologique ne représente $19,25 \%$ des choix relatifs à l'hébergement. Les foyers sont le mode le moins utilisé $(04,71 \%)$ par les élèves. En général, les élèves évoquent leur quête de liberté pour expliquer ou justifier leur choix en faveur du studio. Mais la réalité serait tout autre. En effet, ces différentes situations deviennent prévisibles dès lors que l'on tient compte des données relatives à la répartition des élèves selon la résidence habituelle des parents. Selon ces dernières, $18,71 \%$ seulement des parents 
vivent dans la ville de Danané. Ainsi, 81,29\% des parents vivraient en dehors de celle-ci. Ce qui oblige les élèves à s'orienter plus nombreux vers le studio étant donné la rareté des places dans les foyers. Où vivent les élèves, selon le genre ?

\section{III.2.5 : mode d'hébergement selon sexe comme facteur d'influence sur le rendement scolaire}

Tableau n ${ }^{\circ} 6$ : répartition du mode d'hébergement selon le sexe

\begin{tabular}{|c|c|c|c|c|c|c|c|}
\hline \multirow{2}{*}{$\begin{array}{l}\text { Mode } \\
\text { d'hébergement/Genre }\end{array}$} & \multicolumn{3}{|c|}{ Filles } & \multicolumn{3}{|c|}{ Garçons } & \multirow[b]{2}{*}{ Total } \\
\hline & Eff & $\begin{array}{l}\% \text { obtenu } \\
\text { par le } \\
\text { rapport } \\
\text { vertical }\end{array}$ & $\begin{array}{l}\text { \% obtenu } \\
\text { par le } \\
\text { rapport } \\
\text { horizontal }\end{array}$ & Eff & $\begin{array}{l}\% \text { obtenu } \\
\text { par le } \\
\text { rapport } \\
\text { vertical }\end{array}$ & $\begin{array}{l}\text { \% obtenu } \\
\text { par le } \\
\text { rapport } \\
\text { horizontal }\end{array}$ & \\
\hline En famille & 40 & 25,16 & 39,21 & 62 & 16,71 & 60,78 & 102 \\
\hline Chez un tuteur & 51 & 32,08 & 32,48 & 106 & 28,57 & 67,51 & 157 \\
\hline Dans un foyer & 11 & 06,92 & 44,00 & 14 & 03,77 & 65,00 & 25 \\
\hline Dans un studio & 57 & 35,85 & 23,17 & 189 & 50,94 & 76,82 & 246 \\
\hline Total & \multicolumn{3}{|c|}{159} & \multicolumn{3}{|l|}{317} & 530 \\
\hline
\end{tabular}

Source : enquête du 04 Avril au 04 juin 2019 au lycée moderne de Danané

Le genre n'est pas un critère discriminant dans le choix du mode d'hébergement. Féminin comme masculin vivent majoritairement en studio. Les filles y résident à proportion de 35,85\%, contre 50,94 pour les garçons. Quête de liberté, mais aussi et surtout inexistant d'un mécanisme de tutorat peuvent expliquer la proportion des élèves, indifféremment du genre, à choisir le studio comme mode prioritaire d'hébergement. Quand le Tableau de Bord Vie Scolaire édition 2018, indique que pour l'année scolaire 2018-2019, on a enregistré 1500 cas de grossesse d'élèves, et que cela équivaut à la fermeture virtuelle d'un lycée entier de filles, on peut avoir des appréhensions. L'offre éventuelle de comportements déviants que renferme cette situation, n'est pas à négliger. Où vivent les élèves, par rapport à leurs niveaux d'études ?

\section{III.2.6 : mode d'hébergement par niveaux comme facteur d'influence sur le rendement scolaire}

Tableau n ${ }^{\circ}$ : répartition selon le mode d'hébergement par niveaux d'études

\begin{tabular}{|l|l|l|l|l|l|}
\hline $\begin{array}{l}\text { Mode d'hébergement/niveau d'étude/ } \\
\text { Effectif et Proportion obtenue par le rapport } \\
\text { Vertical et Horizontal }\end{array}$ & $6^{\text {ème }}$ & $5^{\text {ème }}$ & $4^{\text {ème }}$ & $3^{\text {ème }}$ & Total \\
\hline \multirow{3}{*}{ Parent } & 03 & 27 & 49 & 23 & \\
& 02,63 & 18,75 & 35,25 & 17,29 & 102 \\
& 02,94 & 26,47 & 48,04 & 22,55 & \\
Tuteur & 40 & 53 & 28 & 36 & \\
& 35,09 & 46,49 & 20,14 & 27,07 & 157 \\
\hline
\end{tabular}




\begin{tabular}{|l|l|l|l|l|l|}
\hline \multirow{3}{*}{ Foyer } & 07 & 01 & 09 & 08 & \multirow{2}{*}{} \\
& 06,14 & 00,08 & 06,47 & 6,01 & 25 \\
& 28,00 & 04,00 & 36,00 & 32,00 & \\
\hline \multirow{3}{*}{ Studio } & 64 & 63 & 53 & 66 & \\
& 56,14 & 55,26 & 38,12 & 49,62 & 246 \\
\hline Total & 26,01 & 25,06 & 21,54 & 26,82 & \\
\hline
\end{tabular}

Source : enquête du 04 Avril au 04 juin 2019 au lycée moderne de Danané

Tout comme le genre, le niveau d'études est un critère indifférent dans le choix du mode d'hébergement des élèves. Quels que soient leurs niveaux d'études, le studio semble les attirer tous. Les élèves de la classe de $6^{\text {ème }}$ qui représentent $21,50 \%$ de l'échantillon, sont beaucoup plus locataires $56,14 \%$ de studio. Pour leur niveau d'étude et pour l'âge auquel renvoie ce niveau, ce mode d'hébergement inspire naturellement, appréhensions. Il en va de même des élèves de la classe de $5^{\text {ème }}$ qui vivent majoritairement $(56,26 \%)$ dans un studio. Pour ceux des classes de $4^{\text {ème }}$ et de $3^{\text {ème }}$, le studio constitue le point de chute de préférence. Ils y habitent respectivement à $38,12 \%$ et $49,62 \%$. Où vivent les élèves, par rapport à leurs origines sociales.

\section{III.2.7 : mode d'hébergement par profession des parents comme facteur d'influence sur le rendement scolaire}

Tableau ${ }^{\circ} 8$ : répartition selon le mode d'hébergement par profession des parents

\begin{tabular}{|l|l|l|l|l|l|l|}
\hline $\begin{array}{l}\text { Mode } \\
\text { d'hébergement/Profession } \\
\text { des parents-Effectif et } \\
\begin{array}{l}\text { Proportion obtenue par le } \\
\text { rapport Vertical et Horizontal }\end{array}\end{array}$ & Planteur & Ouvrier & Cadre & Commerçant & $\begin{array}{l}\text { Sans- } \\
\text { emploi }\end{array}$ & Total \\
\hline & 21 & 33 & 31 & 05 & 12 & \\
Parent & 06,97 & 37,50 & 42,46 & 45,45 & 21,05 & 102 \\
& 20,58 & 32,35 & 30,39 & 04,90 & 11,76 & \\
\hline \multirow{3}{*}{ Tuteur } & 93 & 16 & 17 & 04 & 27 & \\
& 30,89 & 18,18 & 23,28 & 36,36 & 47,36 & 157 \\
\hline & 59,23 & 10,19 & 10,19 & 02,54 & 17,20 & \\
Foyer & 08 & 08 & 05 & 00 & 04 & \\
& 02,65 & 09,90 & 06,84 & 00,00 & 07,02 & 25 \\
\hline \multirow{3}{*}{ Studio } & 32,00 & 32,00 & 20,00 & 00,00 & 16,00 & \\
& 179 & 31 & 20 & 02 & 14 & \\
\hline Total & 59,46 & 35,22 & 27,39 & 18,18 & 24,56 & 246 \\
\hline
\end{tabular}

Source : enquête du 04 Avril au 04 juin 2019 au lycée moderne de Danané

Les enfants de planteurs ou paysans vivent surtout en studio $59,46 \%$. Les différentes catégories sociales d'élèves vivant dans ces studios, ils représentent l'essentiel ( $72,76 \%$ des locataires). Les enfants d'ouvriers, de 
cadres et de commerçants vivent plutôt respectivement à 37,50\%; 42,46\% et $45,45 \%$ chez leurs parents. Mais de toutes les catégories sociales d'élèves vivant chez les parents, ce sont respectivement les enfants d'ouvriers $(32,35 \%)$ et les enfants de cadres $(30,39 \%)$ qui sont les plus nombreux chez leurs parents. Quand aux enfants des sans-emplois, ils vivent en général $(47,36 \%)$ chez des tuteurs. Le manque de moyens financiers explique probablement cette tendance à utiliser un peu plus l'hospitalité. Où vivent les élèves, par rapport à la résidence habituelle des parents ?

\section{III.2.8: mode d'hébergement par résidence habituelle des parents comme facteur d'influence sur le rendement scolaire}

Tableau $\mathbf{n}^{\circ} \mathbf{9}$ : répartition selon le mode d'hébergement par résidence habituelle des parents

\begin{tabular}{|c|c|c|c|c|}
\hline $\begin{array}{l}\text { Mode } \\
\text { d'hébergement/Résidence } \\
\text { habituelle des parents-Effectif } \\
\text { et Proportion obtenue par le } \\
\text { rapport Vertical et Horizontal }\end{array}$ & $\begin{array}{l}\text { Dans la ville } \\
\text { de Danané }\end{array}$ & $\begin{array}{l}\text { Dans la } \\
\text { Région }\end{array}$ & $\begin{array}{l}\text { Hors de } \\
\text { la Région }\end{array}$ & Total \\
\hline Parent & $\begin{array}{l}90 \\
93,75 \\
88,23\end{array}$ & $\begin{array}{l}09 \\
02,83 \\
08,82\end{array}$ & $\begin{array}{l}03 \\
02,56 \\
02,94\end{array}$ & 102 \\
\hline Tuteur & $\begin{array}{l}02 \\
02,08 \\
01,27\end{array}$ & $\begin{array}{l}103 \\
32,49 \\
65,60\end{array}$ & $\begin{array}{l}52 \\
44,44 \\
33,12\end{array}$ & 157 \\
\hline Foyer & $\begin{array}{l}00 \\
00,00 \\
00,00\end{array}$ & $\begin{array}{l}18 \\
05,67 \\
72,00\end{array}$ & $\begin{array}{l}07 \\
05,98 \\
28,00\end{array}$ & 25 \\
\hline Studio & $\begin{array}{l}04 \\
04,16 \\
01,62\end{array}$ & $\begin{array}{l}187 \\
58,99 \\
76,01\end{array}$ & $\begin{array}{l}55 \\
47,00 \\
22,35\end{array}$ & 246 \\
\hline Total & 96 & 317 & 117 & 530 \\
\hline
\end{tabular}

Source : enquête du 04 Avril au 04 juin 2019 au lycée moderne de Danané

Lorsque la résidence habituelle des parents est dans la ville, naturellement leurs enfants vivent majoritairement chez eux $(93,75)$. Une infirme partie $(06,25 \%)$ relève de situations dont l'explication tiendrait de la paupérisation de certaines couches sociales. Lorsque les parents résident dans la Région, leurs enfants vivent principalement $(58,99 \%)$ en studio et secondairement chez des tuteurs $(32,49 \%)$. De même, lorsque les parents résident hors de la Région, leurs enfants vivent principalement $(47,00 \%)$ en studio et secondairement chez les tuteurs $(44,44 \%)$. 


\section{III.3 : relation entre rendement scolaire et conditions d'hébergement selon la satisfaction des besoins en manuels scolaires}

Tableau $\mathbf{n}^{\circ} \mathbf{1 0}$ : conditions d'hébergement et satisfaction des besoins en manuels scolaires

\begin{tabular}{|l|l|l|l|}
\hline $\begin{array}{l}\text { Conditions } \\
\text { hébergement/Besoins en } \\
\text { manuels scolaire }\end{array}$ & $\begin{array}{l}\text { Elèves vivant sous } \\
\text { l'autorité d'une personne } \\
\text { adulte (Parents et } \\
\text { tuteurs) }\end{array}$ & $\begin{array}{l}\text { Elèves vivant hors de } \\
\text { l'autorité d'une personne } \\
\text { adulte (Foyer et Studio) }\end{array}$ & Total \\
\hline Tous les manuels & 69 & 70 & 139 \\
\hline La moitié des manuels & 176 & 184 & 360 \\
\hline Aucun manuel & 14 & 17 & 31 \\
\hline Total & 259 & 271 & 530 \\
\hline
\end{tabular}

Source : enquête du 04 Avril au 04 juin 2019 au lycée moderne de Danané

A ce niveau de la satisfaction des besoins en manuels scolaires, le test d'indépendance des variables nous donne ceci : dans les hypothèses de départ, l'hypothèse nulle ( $\mathrm{H}$ 0) signifie qu'il n'y a pas d'influence entre le mode d'hébergement et la satisfaction des besoins en manuels scolaires. L'hypothèse alternative (H1) signifie que le mode d'hébergement influence la satisfaction des besoins en manuels scolaires.

$x_{\text {cal }}^{2}=0,2$

$\mathrm{Ddl}=2$ avec un niveau d'erreur égal à $5 \%(0,05), \quad x_{l u}^{2}=5,99$

$x_{c a l}^{2}<x_{l u}^{2}$. L'hypothèse nulle est acceptée. Il n'y a donc pas d'influence entre le mode d'hébergement et la satisfaction des besoins en manuels scolaires.

\section{III.4 : relation entre rendement scolaire et conditions d'hébergement selon la satisfaction des besoins alimentaires}

Tableau $\mathbf{n}^{\circ} 11$ : conditions d'hébergement et satisfaction des besoins alimentaires

\begin{tabular}{|l|l|l|l|}
\hline $\begin{array}{l}\text { Conditions } \\
\text { hébergement/Besoins } \\
\text { alimentaires }\end{array}$ & $\begin{array}{l}\text { Elèves vivant sous } \\
\text { l'autorité d'une personne } \\
\text { adulte (Parents et tuteurs) }\end{array}$ & $\begin{array}{l}\text { Elèves vivant hors de } \\
\text { l'autorité d'une } \\
\text { personne adulte (Foyer } \\
\text { et Studio) }\end{array}$ & Total \\
\hline Trois repas & 87 & 34 & 121 \\
\hline Deux repas & 127 & 185 & 312 \\
\hline Un repas & 45 & 52 & 97 \\
\hline Total & 259 & 271 & 530 \\
\hline
\end{tabular}

Source : enquête du 04 Avril au 04 juin 2019 au lycée moderne de Danané

Dans les hypothèses de départ, l'hypothèse nulle $(\mathrm{H}$ 0) signifie qu'il n'y a pas d'influence entre le mode d'hébergement et l'alimentation. L'hypothèse (H1) signifie que le mode d'hébergement influence l'alimentation.

$x_{\text {cal }}^{2}=34,0$

$\mathrm{Ddl}=2$ avec un niveau d'erreur égal à $5 \%(0,05), \quad x_{l u}^{2}=5,99$ 
$x_{c a l}^{2}>x_{l u}^{2}$. L'hypothèse nulle est rejetée. Il y a donc relation entre le mode d'hébergement et satisfaction des besoins alimentation.

\section{III.5 : relation entre rendement scolaire et conditions d'hébergement selon l'organisation de personnelle d'étude}

Tableau n ${ }^{\circ} 12$ : conditions d'hébergement et l'organisation de personnelle d'étude

\begin{tabular}{|l|l|l|l|}
\hline $\begin{array}{l}\text { Conditions } \\
\text { hébergement/Organisation } \\
\text { personnelle }\end{array}$ & $\begin{array}{l}\text { Elèves vivant sous } \\
\text { l'autorité d'une } \\
\text { personne adulte } \\
\text { (Parents et tuteurs) }\end{array}$ & $\begin{array}{l}\text { Elèves vivant hors } \\
\text { de l'autorité d'une } \\
\text { personne adulte } \\
\text { (Foyer et Studio) }\end{array}$ & Total \\
\hline Oui & 144 & 143 & 287 \\
\hline Non & 115 & 128 & 243 \\
\hline Total & 259 & 271 & 530 \\
\hline
\end{tabular}

Source : enquête du 04 Avril au 04 juin 2019 au lycée moderne de Danané

Dans les hypothèses de départ, l'hypothèse nulle $(\mathrm{H} 0)$ signifie qu'il n'y a pas d'influence entre le mode d'hébergement et l'organisation personnelle de l'élève. L'hypothèse $(\mathrm{H} 1)$ signifie que le mode d'hébergement influence l'organisation personnelle de l'élève.

$x_{\text {cal }}^{2}=0,43$

Ddl $=1$ avec un niveau d'erreur égal à $5 \%(0,05), \quad x_{l u}^{2}=3,84$

$x_{c a l}^{2}<x_{l u}^{2}$. L'hypothèse nulle est acceptée. Il n'y a donc pas de relation entre le mode d'hébergement et l'organisation personnelle de l'élève.

\section{III.6 : relation entre rendement scolaire et conditions d'hébergement selon l'utilisation d'un répétiteur}

Tableau $n^{\circ} 13$ : conditions d'hébergement et l'utilisation d'un répétiteur

\begin{tabular}{|l|l|l|l|}
\hline $\begin{array}{l}\text { Conditions } \\
\text { hébergement/Utilisation de } \\
\text { répétiteurs }\end{array}$ & $\begin{array}{l}\text { Elèves vivant sous } \\
\text { l'autorité d'une } \\
\text { personne adulte } \\
\text { (Parents et tuteurs) }\end{array}$ & $\begin{array}{l}\text { Elèves vivant hors de } \\
\text { l'autorité d'une } \\
\text { personne adulte (Foyer } \\
\text { et Studio) }\end{array}$ & Total \\
\hline Oui & 49 & 43 & 92 \\
\hline Non & 210 & 228 & 438 \\
\hline Total & 259 & 271 & 530 \\
\hline
\end{tabular}

Source : enquête du 04 Avril au 04 juin 2019 au lycée moderne de Danané

Dans les hypothèses de départ, l'hypothèse nulle ( $\mathrm{H} 0)$ signifie qu'il n'y a pas d'influence entre le mode d'hébergement et l'utilisation de répétiteur par l'élève. L'hypothèse alternative $(\mathrm{H} 1)$ signifie que le mode d'hébergement influence l'utilisation de répétiteur par l'élève.

$x_{\text {cal }}^{2}=0,86$

Ddl $=1$ avec un niveau d'erreur égal à $5 \%(0,05), \quad x_{l u}^{2}=3,84$

$x_{c a l}^{2}<x_{l u}^{2}$. L'hypothèse nulle est acceptée. Il n'y a donc pas de relation entre le mode d'hébergement et l'utilisation de répétiteur par l'élève. 


\section{III.7 : relation entre rendement scolaire et conditions d'hébergement selon l'affectivité parentale}

Tableau $\mathbf{n}^{\circ} 14$ : conditions d'hébergement et l'affectivité parentale

\begin{tabular}{|l|l|l|l|}
\hline $\begin{array}{l}\text { Conditions } \\
\text { hébergement/Affectivité } \\
\text { parentale }\end{array}$ & $\begin{array}{l}\text { Elèves vivant sous } \\
\text { l'autorité d'une } \\
\text { personne adulte } \\
\text { (Parents et tuteurs) }\end{array}$ & $\begin{array}{l}\text { Elèves vivant hors de } \\
\text { l'autorité d'une } \\
\text { personne adulte (Foyer } \\
\text { et Studio) }\end{array}$ & Total \\
\hline Oui & 254 & 265 & 519 \\
\hline Non & 05 & 06 & 11 \\
\hline Total & 259 & 271 & 530 \\
\hline
\end{tabular}

Source : enquête du 04 Avril au 04 juin 2019 au lycée moderne de Danané

Dans les hypothèses de départ, l'hypothèse nulle ( $\mathrm{H} 0)$ signifie qu'il n'y a pas d'influence entre le mode d'hébergement et le sentiment d'être aimé de ses parents. L'hypothèse alternative (H1) signifie que le mode d'hébergement influence le sentiment d'être aimé de ses parents.

$x_{\text {cal }}^{2}=232$

$\mathrm{Ddl}=1$ avec un niveau d'erreur égal à $5 \%(0,05), \quad x_{l u}^{2}=3,84$

$x_{c a l}^{2}>x_{l u}^{2}$. L'hypothèse nulle est rejetée. Il y a relation entre le mode d'hébergement et le sentiment d'être aimé de ses parents.

\section{III.8 : relation entre rendement scolaire et conditions d'hébergement selon l'environnement humain immédiat}

Tableau $\mathbf{n}^{\circ} \mathbf{1 5}$ : conditions d’hébergement et environnement humain immédiat

\begin{tabular}{|l|l|l|l|}
\hline $\begin{array}{l}\text { Conditions } \\
\text { hébergement/Environnement } \\
\text { humain immédiat }\end{array}$ & $\begin{array}{l}\text { Elèves vivant sous } \\
\text { l'autorité d'une personne } \\
\text { adulte (Parents et tuteurs) }\end{array}$ & $\begin{array}{l}\text { Elèves vivant hors de } \\
\text { l'autorité d'une personne } \\
\text { adulte (Foyer et Studio) }\end{array}$ & Total \\
\hline Oui & 251 & 246 & 497 \\
\hline Non & 08 & 25 & 33 \\
\hline Total & 259 & 271 & 530 \\
\hline
\end{tabular}

Source : enquête du 04 Avril au 04 juin 2019 au lycée moderne de Danané

Dans les hypothèses de départ, l'hypothèse nulle ( $\mathrm{H} 0)$ signifie qu'il n'y a pas d'influence entre le mode d'hébergement et l'idée que se fait l'élève sur la qualité de ses rapports avec son environnement humain immédiat. L'hypothèse (H 1) signifie que le mode d'hébergement influence l'idée que se fait l'élève sur la qualité de ses rapports avec son environnement humain immédiat.

$x_{\text {cal }}^{2}=194$

$\mathrm{Ddl}=1$ avec un niveau d'erreur égal à $5 \%(0,05), \quad x_{l u}^{2}=3,84$

$x_{c a l}^{2}>x_{l u}^{2}$. L'hypothèse nulle est rejetée. Il y a donc une relation entre le mode d'hébergement et l'idée que se fait l'élève sur la qualité de ses rapports avec son environnement humain immédiat. 


\section{III.9 : relation entre rendement scolaire et conditions d'hébergement par niveaux d'études}

\section{III.9.1: relation entre rendement scolaire et conditions d'hébergement pour le niveau $6^{\text {ème }}$}

Tableau $n^{\circ} 16$ : conditions d'hébergement et rendement scolaire-niveau $6^{\text {ème }}$

\begin{tabular}{|l|l|l|l|}
\hline $\begin{array}{l}\text { Conditions } \\
\text { hébergement/Rendement } \\
\text { scolaire }\end{array}$ & $\begin{array}{l}\text { Elèves vivant sous } \\
\text { l'autorité d'une } \\
\text { personne adulte } \\
\text { (Parents et tuteurs) }\end{array}$ & $\begin{array}{l}\text { Elèves vivant hors de } \\
\text { l'autorité d'une } \\
\text { personne adulte (Foyer } \\
\text { et Studio) }\end{array}$ & Total \\
\hline Non redoublant & 248 & 239 & 487 \\
\hline Redoublant & 23 & 20 & 43 \\
\hline Total & 271 & 259 & 530 \\
\hline
\end{tabular}

Source : enquête du 04 Avril au 04 juin 2019 au lycée moderne de Danané

Dans les hypothèses de départ, l'hypothèse nulle ( $\mathrm{H} 0)$ signifie qu'il n'y a pas d'influence entre le mode d'hébergement et le rendement scolaire de classe de $6^{\text {ème.. }}$ L'hypothèse alternative $\left(\begin{array}{ll}\mathrm{H} & 1\end{array}\right)$ signifie que le mode d'hébergement influence le rendement scolaire de classe de $6^{\text {ème. }}$ $x_{\text {cal }}^{2}=0,101$

Avec un niveau d'erreur égal à $5 \%(0,05), \quad x_{l u}^{2}=3,84$

$x_{c a l}^{2}<x_{l u}^{2}$. L'hypothèse nulle $(\mathrm{H} 0)$ est acceptée. L'hypothèse alternative $(\mathrm{H}$ 1) est rejetée. Il n'y a donc pas relation entre les variables. On peut conclure que les conditions d'hébergement n'influencent pas le rendement scolaire des classes de $6^{\text {ème }}$. On observe qu'au niveau du taux de redoublement en se référant au tableau on voit que 23 redoublants sont des élèves qui vivent sous l'autorité de personnes adultes et que 20 autres doublant sont des élèves, affranchis de cette autorité. Les premiers ont un taux de redoublement de $53,5 \%$ et les seconds, $46,5 \%$. Un peu plus de la moitié des élèves qui redoublent la classe de $6^{\text {ème }}$, sont des qui vivent sous l'autorité des adultes.

\section{III.9.2 : relation entre rendement scolaire et conditions d'hébergement pour le niveau $5^{\text {ème }}$.}

Tableau $\mathbf{n}^{\circ} 17$ : conditions d'hébergement et rendement scolaire-niveau $5^{\text {ème }}$

\begin{tabular}{|l|l|l|l|}
\hline $\begin{array}{l}\text { Conditions } \\
\text { hébergement/rendement } \\
\text { scolaire }\end{array}$ & $\begin{array}{l}\text { Elèves vivant sous } \\
\text { l'autorité d'une } \\
\text { personne adulte } \\
\text { (Parents et tuteurs) }\end{array}$ & $\begin{array}{l}\text { Elèves vivant hors de } \\
\text { l'autorité d'une } \\
\text { personne adulte } \\
\text { (Foyer et Studio) }\end{array}$ & Total \\
\hline Non redoublant & 184 & 197 & 381 \\
\hline Doublant & 16 & 19 & 35 \\
\hline Total & 200 & 216 & 416 \\
\hline
\end{tabular}

Source : enquête du 04 Avril au 04 juin 2019 au lycée moderne de Danané

Dans les hypothèses de départ, l'hypothèse nulle ( $\mathrm{H} 0)$ signifie qu'il n'y a pas d'influence entre le mode d'hébergement et le rendement scolaire de 
classe de $5^{\text {ème. }}$ L'hypothèse alternative (H1) signifie que le mode $\mathrm{d}$ 'hébergement influence le rendement scolaire de classe de $5^{\text {ème. }}$

$x_{\text {cal }}^{2}=0,125$

Avec un niveau d'erreur égal à $5 \%(0,05), \quad x_{l u}^{2}=3,84$

$x_{c a l}^{2}<x_{l u}^{2}$. L'hypothèse nulle $(\mathrm{H} 0)$ est acceptée. L'hypothèse alternative $(\mathrm{H}$ 1) est rejetée. Il y a donc indépendance entre les variables. On peut conclure que les conditions d'hébergement n'influencent pas le rendement scolaire des classes de $5^{\text {ème }}$. On observe qu'au niveau du taux de redoublement en se référant au tableau on voit que 17 redoublants sont des élèves qui vivent sous l'autorité de personnes adultes et que 18 autres redoublants sont des élèves, affranchis de cette autorité. Les premiers ont un taux de redoublement de $46 \%$ et les seconds, 54\%. Un peu plus de la moitié des élèves qui redoublent la classe de $5^{\text {ème }}$, sont des élèves qui sont affranchis de l'autorité des adultes.

\section{III.9.3 : relation entre rendement scolaire et conditions d'hébergement pour le niveau $4^{\text {ème }}$}

Tableau $\mathbf{n}^{\circ} 18$ : conditions d'hébergement et rendement scolaire-niveau $4^{\text {ème }}$

\begin{tabular}{|l|l|l|l|}
\hline $\begin{array}{l}\text { Conditions } \\
\text { hébergement/rendement } \\
\text { scolaire }\end{array}$ & $\begin{array}{l}\text { Elèves vivant sous } \\
\text { l'autorité d'une } \\
\text { personne adulte (Parents } \\
\text { et tuteurs) }\end{array}$ & $\begin{array}{l}\text { Elèves vivant hors de } \\
\text { '’autorité d'une } \\
\text { personne adulte (Foyer } \\
\text { et Studio) }\end{array}$ & Total \\
\hline Non redoublant & 105 & 100 & 205 \\
\hline Redoublant & 32 & 36 & 68 \\
\hline Total & 137 & 136 & 273 \\
\hline
\end{tabular}

Source : enquête du 04 Avril au 04 juin 2019 au lycée moderne de Danané

Dans les hypothèses de départ, l'hypothèse nulle $(\mathrm{H} 0)$ signifie qu'il n'y a pas d'influence entre le mode d'hébergement et le rendement scolaire de classes de $4^{\text {ème. }}$ L'hypothèse alternative $(H \quad 1)$ signifie que le mode d'hébergement influence le rendement scolaire.

$x_{\text {cal }}^{2}=0,3133$

Avec un niveau d'erreur égal à $5 \%(0,05), \quad x_{l u}^{2}=3,84$

$x_{c a l}^{2}<x_{l u}^{2}$. L'hypothèse nulle $(\mathrm{H} 0)$ est acceptée. L'hypothèse alternative $(\mathrm{H}$ 1) est rejetée. Il y a donc indépendance entre les variables. On peut conclure que les conditions d'hébergement n'influencent pas le rendement scolaire des classes de $4^{\text {ème }}$. On observe qu'au niveau du taux de redoublement en se référant au tableau on voit que 32 redoublants sont des élèves qui vivent sous l'autorité de personnes adultes et que 36 autres redoublant sont des élèves qui vivent affranchis de cette autorité. Les premiers ont un taux de redoublement de $47,05 \%$ et les seconds, 52,94\%. Un peu plus de la moitié des élèves qui redoublent la classe de $4^{\text {ème}}$, sont des qui sont affranchis de l'autorité des adultes. 


\section{Discussion, esquisses de solution et conclusion}

Nous avons prévu d'enquêter sur 30\% de l'effectif du premier cycle, soit 562 élèves avec $1 / 6$ pour les élèves qui habitent chez leurs parents soit 94 , $1 / 6$ pour ceux qui habitent chez des tuteurs soit $94,1 / 3$ pour les pensionnaires des foyers soit 187 et $1 / 3$ pour les locataires de studio soit 187. Cela aurait permis une meilleure structuration de l'échantillonnage. La difficulté est que la plupart des clients des foyers de la ville sont des élèves des établissements scolaires privés. Autant cette situation est gênante, autant elle est intéressante du point de vue de l'analyse psychosociologique. En effet, les parents d'élèves qui ont intériorisé le fait que la scolarisation et la réussite scolaire de l'enfant ont un coût, acceptent plus facilement d'affronter le coût des mesures d'accompagnement, tel que l'hébergement de leur enfant. En revanche, les parents habitués à la gratuité de la scolarité de leur enfant (le principal) rechignent à engager des frais pour son hébergement (l'accessoire). Si le principal est acquis à titre gratuit, pourquoi l'accessoire le serait à titre onéreux, pourrait-on se demander. Dans tous les cas, les certitudes de départ ont été vite bousculées par les réalités du terrain. Sur plusieurs plans, les résultats de l'étude diffèrent de ceux de Bastin et Roosen, 1990, de Ballion, 1993, de Blanchard, Casagrande et McCulloch, 1994 , de Griffaton, 1995, de Best, 1997 et de Bautier et Rochex, 1998, qui pour eux, les conditions de vie des élèves sans conditions ont une influence sur la réussite scolaire. Au plan de la satisfaction des besoins de l'élève, si le mode d'hébergement n'a aucune incidence sur la possession des manuels scolaires et qu'il n'en a que très faiblement sur la ration alimentaire journalière de l'élève, c'est probablement, les mécanismes pour satisfaire ces besoins sont à la portée de tous. En ce qui concerne le manuel scolaire, le développement des moyens de duplication et la tendance de plus en plus forte chez les enseignants à confectionner des brochures sont des facteurs qui démocratisent son accès. L'alimentation relève quant à elle, d'un besoin biologique de premier ordre, qu'aucun élève ne saurait sacrifier. Les ressources financières tirées de l'exécution des contrats de travaux champêtres par les élèves du lycée, apportent un début d'explication. Au plan de l'organisation du travail et en dehors des heures de classe, ni l'organisation personnelle de l'élève, ni l'appui de répétiteurs ne sont influencés par le mode d'hébergement. Il semble que le nivellement culturel de la population explique cette situation. En effet, quelles que soient, les différentes catégories d'élèves vivants sous l'autorité d'une personne adulte (parents ou tuteurs) et ceux vivants hors de l'autorité d'une personne adulte (foyer ou studio) vivent probablement, dans une culture ambiante qui ne laisse pas assez de places aux particularités. Au plan du facteur affectif, le mode d'hébergement détermine assez fortement le sentiment d'être aimé de ses parents. Il détermine dans les mêmes termes, l'idée que l'élève se fait de la qualité de ses rapports avec son environnement humain immédiat. Ces 
situations montrent assez bien que l'élément affectif est le maillon le plus réceptif chez l'élève. Au plan du rendement scolaire, le logement de l'élève est indifférent à son rendement scolaire. Quel que soit le niveau d'étude (6 ${ }^{\text {ème }}$, $5^{\text {ème }}$ et $\left.4^{\text {ème }}\right)$, les conditions d'hébergement ne semblent pas influencer le rendement scolaire. De tels résultats peuvent en effet surprendre, lorsque les pronostics de départ sont sous-tendus par un substrat déterministe. Toutefois, une analyse plus fine de ces données pourrait aider à la compréhension de ceux-ci. Il ne faut en effet, pas perdre de vue que l'étude s'est déroulée en zone semi-rurale. Une semi-ruralité à laquelle la localisation du lycée dans la ville, ne change pas grand-chose. Au-delà du critère géographique, il y a un ensemble d'éléments qui situent mieux, sur la question. D'abord, c'est l'origine sociale des élèves. L'étude montre que la catégorie socioprofessionnelle ayant le plus d'enfants dans l'établissement, est celle des planteurs ou paysans. Les enfants de planteurs ou paysans représentent $64,15 \%$ de la population scolaire. Une telle configuration démographique entretien nécessairement des façons de penser et d'agir. Ensuite, vient la résidence habituelle des parents. Ceux-ci résident habituellement et de façon constante, dans des villages et campements satellites de la ville. Ils y sont à $59,81 \%$. Enfin, il y a le dépaysement. Cette désorientation du sujet qui lui crée préjudice, est absente dans le cas de l'étude. L'inventaire des conditions de vie des élèves du lycée, renvoie sensiblement aux conditions normales de vie dans les campements. De sorte que, la vie de l'élève en ville, n'est que le prolongement de celle qu'il a au village ou au campement. La stratégie mise en place par les élèves pour combler leur déficit d'argent de poche corrobore cette idée. Car quelle que soit leur origine sociale, ils sont concernés par les travaux champêtres (les enfants de planteur à $62,64 \%$, les enfants d'ouvrier à $47,25 \%$, les enfants de cadre à 57,53\%, les enfants de commerçant à 72,72\% et les enfants de sans emploi à 33,33\%). Ainsi, se sont tous les enfants qui se constituent, à des proportions différentes, en main d'œuvre agricole pour pourvoir à leurs besoins. Ces quelques éléments montrent assez bien que nous sommes en milieu rural et que tout ce qui pourrait paraitre précaire voire inhibiteur fait partie d'un ensemble dont l'élève s'est déjà bien adapté. A cela, il convient d'ajouter d'autres facteurs parmi lesquels il y a, la satisfaction des besoins alimentaires. L'étude montre qu'environ $59 \%$ des élèves ont une ration alimentaire journalière de deux repas par jour, ce qui permet de s'assurer une résistance physique. Il y a également le facteur affectif qui joue favorablement dans l'équilibre des élèves. Dans une proportion très importante $94,71 \%$ ces derniers se sentent aimés de leurs parents et disent 86,98\% avoir de bonnes relations avec leur environnement humain immédiat.

En dehors de toute recherche de corrélation, le taux de redoublement entre élèves vivants sous l'autorité de personnes adultes et élèves affranchis de cette autorité peut être un critère de comparaison. Cette étude montre que 
sur ce plan le fait d'être un élève de la classe de $6^{\text {ème }}$, vivant en dehors de l'autorité d'une personne adulte n'expose pas plus au redoublement. Par contre, en $5^{\text {ème }}$ et $4^{\text {ème }}$, l'étude montre que le fait d'être un élève vivant en dehors de l'autorité d'une personne adulte, expose un peu plus au redoublement. Ces situations sont moins liées aux conditions d'hébergement qu'au développement intellectuel, affectif et moral de l'adolescent. En effet, l'âge légal de la scolarisation en Côte d'Ivoire est fixé à partir de 6 ans dans le primaire. Mais dans les faits, les problèmes d'accès à l'école repoussent toujours cet âge théorique à 8 voire 15 ans en zone rurale. Ainsi, les élèves de $5^{\text {ème }}$ et $4^{\text {ème }}$ ont un âge qui oscille entre 14 et 16 ans selon les cas. Ce sont justement des seuils autour desquels des adolescents qui, ont en prime une liberté quasi totale, peuvent innocemment sacrifier d'importantes parcelles d'une vie. L'étude montre que, dans deux cas sur trois, les taux de redoublements sont relativement plus élevés chez les élèves affranchis de l'autorité des personnes adultes, sans doute en raison de leur adolescence. Toutefois, la recherche de corrélation montre que le rendement des élèves et les modalités d'hébergement, sont des variables qui s'ignorent. Ces conclusions ne sont pas celles des études d'Adda, 1997, de Bih, 1997, de Dedy, Bih et Koné, 1997, de Koné, 1998 et Kouadio, 2015 et 2017. L'harmonie entre la vie que leur imposent leur origine sociale et les conditions de vie des élèves en ville, explique cette absence de différenciation. Si pour l'instant, les conditions de vie ne semblent pas avoir d'impact sur les rendements des élèves, il est à craindre que dans un avenir proche, cette situation soit forclose. Les convulsions orchestrées par les élèves en vue de se précipiter les différents congés scolaires ne sont-ils pas le signe que la grande liberté est en train de changer les comportements en zone rurale en particulier le lycée moderne de Danané ? Cette préoccupation, beaucoup d'auteurs la partage dans le monde même si les réalités géographiques diffèrent d'un pays à autres (Clerget, 2000 ; Flessas et Lussier, 2001 ; Beaumont, 2003 ; Boutin, et Daneau, 2004 ; Crosnoe, et Elder, 2004 ; Goupil, 2004 ; Asdih, et GezM'bembo, 2005 ; Forget, Schuessler, Paquet, et Giroux, 2005; Fourez, 2006 et Coudronnière, Bacro, Guimard et Florin, 2015).

Voila pourquoi cette situation appelle à des interventions multiformes à plusieurs niveaux. Mais deux seront retenus, en raison de leur pertinence et de leur faisabilité pour l'étude. Au niveau des parents d'élèves, il s'agit surtout de renforcer la mobilisation communautaire autour de l'école, afin de mieux comprendre ses missions et son fonctionnement. Ce qui permettra de bien identifier le type d'assistance à apporter aux élèves. Car, la réussite de ceuxci repose, au-delà de leur effort et de leur travail scolaire, sur l'appui que leur apportent leurs parents. Au niveau de l'État, il convient de reprendre dans des conditions nouvelles, la construction d'établissements à régime internat. À défaut, d'initier une politique d'incitation des collectivités décentralisées, à la 
construction d'auberges pour élèves. Cela assurera un meilleur encadrement à ces adolescents et leur garantira, de meilleures conditions de vie et de travail. Dans le même ordre d'idée, l'Etat devrait réorganiser le domaine des foyers privés. Il pourrait par exemple réglementer les aspects techniques par l'élaboration de normes spécifiques de construction des édifices abritant les foyers, réglementer le fonctionnement de ces foyers, accorder des facilités d'ordre fiscal aux operateurs immobiliers du domaine. Enfin, pour mettre un terme au phénomène des élèves voyageurs, affectés et orientés loin de la résidence des parents, les établissements de proximité devraient être réalisés. $\mathrm{Au}$ terme de cette étude, nous pouvons affirmer que les élèves échappent de plus en plus à l'autorité des adultes du fait de l'inexistence de structures d'hébergement. Nous pouvons également soutenir que l'hospitalité continue d'être un moyen d'accès à l'école, comme elle l'a été depuis le début des indépendances en Afrique et plus particulièrement en Côte d'Ivoire.

\section{References:}

1. Adda, N. (1997). Réflexion sur l'échec de l'école ou l'échec à l'école. Revue Ivoirienne des Sciences de l'Education. Pp 63-75.

2. Amigues, R et Zerbato-Poudou, M. T. (2000). Comment l'enfant devient élève. Les apprentissages à l'école maternelle. Paris : Retz.

3. Asdih, C et Gez-M'bembo, D. (2005). Le décrochage scolaire : la problématique et analyse thématique du discours de décrocheurs français. In D. Zay (dir.), Prévenir l'exclusion scolaire et sociale des jeunes (p. 77-101). Paris: Presses universitaires de France.

4. Audas, R et Willms, D. (2001). Engagement and dropping out of school: A life-course perspective. Applied Research Branch: Hull. 62 p.

5. Baker, J. A. (2006). Contributions of teacher-child relationships to positive school adjustment during elementary school. Journal of School Psychology, 44(3), 211-229.

6. Baker, J., L. Dilly, J. Aupperlee et Patil. S. (2003). The developmental context of school satisfaction: Schools as psychologically healthy environments. School Psychology Quarterly 2 (18), 206.

7. Ballion, R. (1993). Le lycée, une cité à construire. Paris : Hachette. $\mathrm{Pp}$ 05-19.

8. Bastin, G et Roosen, A. (1990). L'école malade de l'échec. Paris : Éditions Universitaires.

9. Bautier, E et Rochex, J-Y. (1998). L'expérience scolaire des nouveaux lycéens. Démocratisation ou massification? Paris: Armand Colin.

10. Beaumont, C. (2003). Les effets d'un programme adapté de médiation par les pairs auprès d'élèves en troubles de comportement. Copyright Claire Beaumont, Doctorat en psychopédagogie. (Dans Les effets d'un 
programme adapté de médiation par les pairs auprès d'élèves en trouble de comportement).

11. Beaumont, C., Royer, E., Bertrand, R et Bowen, F. (2003). La médiation par les pairs et les élèves en troubles du comportement. Revue de Psychoéducation, 32, 79-103.

12. Bee, H. (1997). Les âges de la vie: Psychologie du développement humain. Saint-Laurent : Renouveau Pédagogique.

13. Bee, H et Boyd, D. (2003). Les âges de la vie: Psychologie du développement humain ( $2^{\mathrm{e}} \mathrm{E} d$.) Éditions du renouveau pédagogique, 137-140

14. Berndt, T. J., Hawkins, J. A et Jiao, Z. (1999). Influences of friends and friendships on adjustment to junior high school. Merrill-Palmer Quartely, 45, 13-41.

15. Bertrand, Y (1990). Théories contemporaines de l'éducation. Les éditions Agence d'Arc, Ottawa p. 16-24.

16. Best, F. (1997). L'échec scolaire. Paris : Presses universitaires de France.

17. Bih, E. (1997). Conditions socio-économiques des parents et stratégie d'encadrement extrascolaire des élèves du primaire. Revue Ivoirienne des Sciences de l'Education. Pp 27-40.

18. Blanchard, F., Casagrande, E et McCulloch, P. (1994). Echec scolaire. Nouvelles perspectives systémiques. Paris : ESF.

19. Bonnéry, S. (2008). Échec scolaire. In A. Van Zanten (dir.), Dictionnaire de l'éducation. Paris : Presses universitaires de France. Pp 173-175

20. Boutin, G. et Daneau, C. (2004). Réussir: prévenir et contrer l'échec scolaire. Montréal: Éditions Nouvelles.

21. Bowen, F et Desbiens, N. (2002). La prévention des conduites violentes en milieu scolaire: Évaluer pour développer de meilleures pratiques. Bulletin du CRIRES, mai- juin 2002, 17-24.

22. Braun, M.J.C. (2000). Neuropsychologie du Développement. Paris : Médecine - Sciences Flammarion.

23. Chariot, B. (2000). Le rapport au savoir en milieu populaire: « apprendre à l'école » et « apprendre la vie ». Revue ville-écoleintégration-enjeux, 123, 56-63.

24. Clerget, S. (2000). Adolescents, la crise nécessaire. Paris: Fayard.

25. Coudronnière, C., F. Bacro, P. Guimard et A. Florin (2015). Quelle conception de la qualité de vie et du bien-être chez des enfants de 5 à 11 ans ? Enfance (02), 225-243

26. Corsini, J. Raymond. (1984). Encyclopedia of Psychology. (en) Raymond J. Corsini, Danny Wedding, Frank Dumont, Current 
psychotherapies, Wadsworth Publishing Co Inc, 14 mars 2010, 9 th éd., 640 p.

27. Crosnoe, R et Elder, G. H. (2004). Family dynamics, supportive relationships, and educational resilience during adolescence. Journal of Family Issues, 25(5), 571-602.

28. Crahay, M. (1996). Peut-on lutter contre l'échec scolaire? Bruxelles : De Boeck, Larcier.

29. Currie, C., C. Zanotti, A. Morgan, D. Currie, M. de Looze, C. Roberts, O. Samdal, O. Smith et V. Barnekow (2012). Social determinants of health and well-being among young people. health behaviour in schoolaged children (hbsc) study : international report from the 2009/2010 survey. Technical report, WHO Regional Office for Europe. Copenhagen, Health Policy for Children and Adolescents, No. 6.

30. Debarbieux, E., N. Anton, R. Astor et R. Benbenishty (2012). Le Climat scolaire : définition, effets et conditions d'amélioration. Paris.

31. Dedy, S., Bih, E et Koné, R. (1997). Etude des déterminants familiaux de la scolarisation des filles et des enfants en zones de sousscolarisation en Côte d'Ivoire, ROCARE, Abidjan

32. Destexhe, A., Vandenberghe, V et Vlaeminck, G. (2004). L'école de l'échec : comment la réformer? Du pédagogisme à la gouvernance. Bruxelles : Labor.

33. Dictionnaire. (2018). Le petit Larousse illustré. Paris : édition du bicentenaire de pierre Larousse.

34. Diehl, D. S., Lemerise, E. A., Caverly, S. L., Ramsay, S et Roberts, J. (1998). Peer relations and school adjustment in ungraded primary children. Journal of Educational Psychology, 90, 506-515.

35. Doray, P., Picard, F., Trottier, C et Groleau, A. (2009). Les parcours éducatifs et scolaires; quelques balises conceptuelles. Montréal: Fondation canadienne des bourses d'études du millénaire.

36. Doré, F. Y (1986). L'apprentissage : une approche psychoÉthologique. Le conditionnement classique (Chapitre 6). p. 121-172.

37. Dubé, L. (1996). Psychologie de l'apprentissage, $3^{\mathrm{e}}$ éd. Perspective théorique (Chapitre 14). p. 215-233

38. Duru-Bellat, M. (2002). Les inégalités sociales à l'école. Genèse et mythes. Paris: PUF. Fischer, G.N. (2014). Psychologie sociale de l'environnement. Paris, 2é édition mise à jour, DUNOD.

39. Fiske, E. B. (dir.) (1998). Occasions perdues: quand l'école faillit à sa mission. L'abandon et le redoublement dans l'enseignement primaire. Paris : UNESCO.

40. Flessas, J et Lussier, F. (2001). Neuropsychologie de l'enfant, Troubles développementaux et de l'apprentissage. Paris: Dunod. 
41. Fuchs, D et Fuchs, L. S. (1998). Researchers and teachers working together to adapt instruction for diverse learners. Learning Disabilities Research \& Practice, 13, 126-137.

42. Forget, J., Schuessler, K., Paquet, A et Giroux, N. (2005). Analyse appliquée $\mathrm{du}$ comportement et intervention comportementale intensive. Revue québécoise de psychologie, 26(3), 29-42.

43. Fourez, G. (2006). Éduquer. Enseignants, élèves, écoles, éthiques, sociétés (3e édition). Bruxelles : De Boeck, Larcier.

44. Goupil, G. (2004). Plans d'intervention, de services et de transition. Montréal : Gaëtan Morin éditeur.

45. Griffaton, C. (1995). L'hébergement des élèves en internat : le cas de l'Enseignement Technique. Rapport reprographie. Paris

46. Guimard, P., F. Bacro et A. Florin (2014). Évaluer la satisfaction scolaire et le bien-être des élèves à l'école et au collège. Paris. Dans P. Guimard et C. Sellenet (Eds.). Evaluation des besoins des enfants et qualité de vie : regards croisés France-Canada.

47. Guimard, P., F. Bacro, A. Florin, S. Ferrière et T. Gaudonville (2017). Évaluation du bien-être perçu des élèves : étude longitudinale à l'école élémentaire et au collège. Recherche complémentaire (BEScol2).Paris.

48. Guimard, P., F. Bacro, A. Florin, S. Ferrière, T. Gaudonville et H. Thanh-Ngo (2015). Le bien-être des élèves à l'école et au collège. Validation d'une échelle multidimensionnelle, analyses descriptives et différentielles. Éducation \& formations, 88-89, 163-184.

49. Hébert, J., Boissé, I et Audet, J. (1998). La médiation par les pairs au primaire : Guide d'animation. Montréal : Centre Mariebourg.

50. Kamanzi, P. C. et Bélanger, P. W. (2003). Les redoublements dans l'enseignement primaire en Afrique subsaharienne : problèmes et stratégies. In F. Larose (éd.), Difficultés d'adaptation sociale ou scolaire et intervention éducative (p. 40-62). Sherbrooke: Éditions du CRP.

51. Koné, R. (1998). Origine sociale et performance scolaire, notes en Français de collégiens Abidjanais. Revue Ivoirienne des Sciences de L'Education. Abidjan pp 104-113.

52. Kouadio, K.A. (2015). Investissement parental et Performances scolaires des élèves des Ecoles Primaires Publiques et Privées du District d'Abidjan: cas des groupes scolaires Saint Jean Bosco de Treichville et Plateau Dokui. Thèse Unique de Doctorat. Université Alassane Ouattara de Bouaké.

53. Kouadio, K.A. (2017). Types de familles, autorité parentale et trajectoire scolaires des élèves de CM2 : Pistes pour comprendre. Revue Ivoirienne des Sciences de l'Education. Pp 8-20. 
54. Koudou, K.R. (2006). Urbanisation et violence des jeunes de rue à Abidjan: quelle validité pour la théorie de l'espace défendable en criminologie? Revue Africaine de Criminologie, violences et insécurité dans la cité, (3), 8- 33.

55. L'Abbé, Y et Morin, D. (2001). Comportements agressifs et retard mental : Compréhension et intervention ( $2^{\mathrm{e}}$ éd.). Eastman : Édition Behaviora

56. Lange, M.-F. (2003). École et mondialisation Vers un nouvel ordre scolaire? Cahiers d'études africaines, 1(2), 143-166.

57. Lange, M.-F. (2007). Espaces scolaires en Afrique francophone. Ethnologie française, 4 (37), 639-645.

58. Lautrey, J. (1980). Classe sociale, milieu familial et Intelligence. Paris : PUF.

59. Leroi, L. (2007). Des garçons et des cirques. "Mineurs délinquants en Centre Educatif Renforcé (CER).

60. Lovaas, I.O. (1987). Behavioral treatment and normal educational and intellectual Functioning in Young Autistic Children. Journal of Consulting and Clinical Psychology, 55(1), 3-9.

61. Maccoby, E. E et Martin, J. A. (1983). Socialization in the context of the family: Parent-child interaction. Handbook of Child Psychology, 4, $1-101$

62. Magerotte, G et Rogé, B. (2004). Intervention précoce en autisme: un défi pour les praticiens. L'évolution psychiatrique, 69, 579-588.

63. McLoyd, V. C. (1998). Socioeconomic disadvantage and child development. American Psychologist, 53(2), 185-204.

64. Merle, P. (2005). L'élève humilié. L'école, un espace de non-droit? Paris: PUF

65. Merle, P. (2006). À quoi servent les notes? Sciences Humaines, Horssérie spécial, 5, novembre-décembre, 56-59.

66. Millet, M. et Thin, D. (2005). Ruptures scolaires. L'école à l'épreuve de la question sociale. Paris : Presses universitaires de France.

67. Millet, M. et Thin, D. (2007). Scolarités singulières et déterminants sociologiques. Revue Française de pédagogie, 161,41-51.

68. Ministère de l'éducation de Côte d'Ivoire (2017). Rapport des journées nationales de réflexion sur le redoublement. Abidjan : Le Ministère.

69. Ministère de l'éducation de Côte d'Ivoire (2018). Annuaire statistique de l'enseignement du second degré. Abidjan : Le Ministère.

70. Monneret, S et Marc, E. (1996). Apprentissage social et théories cognitives. p.35-56.

71. Moumouni, A. (1998). L'éducation en Afrique. Paris : Présence Africaine. 
72. Morin, E.J. (1996). Les psychologies au travail. Les opérations mentales. p. 97-100.

73. Noumba, I. (2008). Un profil de l'abandon scolaire au Cameroun. Revue d'économie du développement, 1 (22), 37-62.

74. Ormrod, J. E. (1995). Human learning 2nd ed. Operant conditionning (Chapitre 4).p.46-76.

75. Pianta, R. C. (1999). Enhancing relationships between Teachers and Children. Washington, DC: American Psychological Association.

76. Rondeau, N., Bowen, F et Bélanger, J. (1999). Évaluation d'un programme de promotion de la conduite pacifique en milieu scolaire primaire: Rapport final. Montréal: Centre Mariebourg.

77. Schoon, I., Parsons, S et Sacker, A. (2004). Socioeconomic adversity, educational resilience, and subsequent levels of adult adaptation. Journal of Adolescent Research, 19(4), 383-404.

78. Urdan, T et Schoenfelder, E. (2006). Classroom effects on student motivation: Goal structures, social relationships, and competence beliefs. Journal of School Psychology, 44 (2), 331-349.

79. Wang, M., Haertel, G et Walberg, H. (1994). Qu'est-ce qui aide l'élève à apprendre ? Vie Pédagogique, 90, 45-49.

80. Wentzel, K. R. (1998). Social relationships and motivation in middle school: The role of parents, teachers, and peers. Journal of Educational Psychology, 90(2), 202-209.

81. Willms, J. D. (2002). Vulnerable Children - Findings from Canada's National Longitudinal Survey of Children and Youth. Alberta, University of Alberta Press. 